\title{
PLACE OF DEATH IN MORECAMBE BAY: PATTERNS AND PREFERENCES FOR PLACE OF FINAL CARE AND DEATH AMONG TERMINALLY ILL CANCER PATIENTS AND THEIR CARERS
}

\author{
C Thomas, Senior Lecturer; SM Morris, Research Associate; AC Gatrell, \\ Director of the Institute for Health Research; Lancaster University. \\ MB McIllmurray, Consultant Medical Oncologist, Royal Lancaster Infirmary
}

\section{INTRODUCTION}

The government's pledge to improve cancer services includes a commitment to high quality palliative care services that are responsive to the individual needs and preferences of people with cancer, their families and carers. One area in which users may have particular preferences concerns the location of terminal care. Existing national research suggests that the majority of cancer patients would prefer to die at home, but only a small proportion of these have their preferences fulfilled $^{(1)}$. There are marked, but largely unexplained, variations in place of death associated with social class and other social variables ${ }^{(2)}$. Morecambe Bay Health Authority (MBHA) identified a particular need to know more about how preferences and services interact to determine the place of final care for people with cancer in the Morecambe Bay area, where there is an atypical distribution of place of death for people with cancer. These factors inspired us to develop a research project to address issues to do with the place of terminal care. It is hoped that the results of the study will inform local planning and also have wider relevance.

\section{BACKGROUND}

The publication of the Policy Framework for Commissioning Cancer Services $^{(3)}$ has initiated a process of designation, development, and accreditation of palliative care services ${ }^{(4,5)}$. In response to this, the NW Region Palliative Care Group embarked on a programme of accreditation of adult palliative care services within the region, including local selfassessments ${ }^{(6)}$. The self-assessment process in the Morecambe Bay area spawned this research project. MBHA has reviewed palliative care services in the district and considered the options for service development ${ }^{(7)}$. One of the issues which has emerged is the marked difference between the MBHA 'place of death' profile for cancer patients as compared with the national one (Figure1). In addition, there are variations in the place of death of cancer patients across the district ${ }^{(8)}$.

The relatively high proportion of hospice deaths among cancer patients in Lancaster (48\%) and Kendal (28\%) stands out in comparison with Furness and South Lakes (23\%) which is nearer the national average of $17 \%$. The MBHA report noted that 'The reasons for these differences need to be understood before any major service changes are planned These figures show that the number of patients dying at home
- Nationally, $55 \%$ of cancer patients die in hospital, $17 \%$ in hospice, and $26 \%$ at home ${ }^{(9)}$

- In MBHA, $34 \%$ of cancer deaths occur in hospital, $36 \%$ in hospice, and less than $20 \%$ at home $\mathrm{e}^{(7)}$

- Of those dying of cancer in MBHA, hospice deaths account for $23 \%$ of deaths in Furness and the South Lakes; $28 \%$ in Kendal; and $48 \%$ in Lancaster. Only $10 \%$ die at home in Morecambe, compared with $16 \%$ in Kendal, $19 \%$ in Lancaster, $29 \%$ in South Lakes, and $24 \%$ in Furness ${ }^{(7)}$

Figure 1 Where do cancer patients die?

is lower than national averages. This may be the result of patient preferences and may reflect the development of hospice services'(7). The 'patterns and preferences' research project has been set up in response to this need for further information identified by $\mathrm{MBHA}^{(7,8)}$.

\section{WHAT IS KNOWN ABOUT CANCER PATIENTS'AND THEIR CARERS' PREFERENCES REGARDING PLACE OF DEATH?}

Policies are replete with statements that services must reflect users' preferences and accommodate choice, but what is known about preferences with regard to place of final care and death, and how does this compare with actual place of death? In an authoritative paper, Higginson ${ }^{(1)}$ reviewed the research evidence on all aspects of palliative care (Figure 2).

- It has been estimated that $50-70 \%$ of cancer patients would prefer to be cared for or to die at home ${ }^{(10,11}$

- As death approaches cancer patients may change their preferences: hospital and home became less preferred and hospice more preferred, although even one week before death $50 \%$ still wished to be cared for at home ${ }^{(12)}$

- There is strong evidence that conventional care alone fails to meet the needs of many patients and families/carers (for relief of pain and other symptoms; practical, social and emotional needs pre- and post-bereavement)

- Relatives bear the brunt of caring for dying cancer patients. Despite preferences to be cared for/to die at home, acute hospital admission in times of crisis is common ${ }^{(13)}$

Figure 2 Where would patients prefer to die? 
A recent study ${ }^{(14)}$ comparing cancer patients who did/did not die at home found that $21 \%$ of patients died at home and $38 \%$ of all patients were reported by bereaved carers to have expressed a preference for place of death, $73 \%$ of whom wanted to die at home. Of those who had expressed a preference for a home death, only $58 \%$ had achieved this. It was concluded that crucial to the achievement of a home death were: recognition of a preference for a home death, the motivation to 'stick it out' at home, and adequate community support to provide the practical means to fulfill the preference. Difficulties in the final stages of the patient's life can have a lasting effect on carers and those close to the patient, who often carry the burden of care ${ }^{(15)}$. Another recent study in Doncaster Health Authority found statistically significant evidence that place of death is associated with social class, highlighting issues of equity of access to services; the authors conclude that more research is needed into the distribution of preference for place of death among cancer patients ${ }^{(16)}$. Other research evidence indicates that the pattern of place of death also varies with age and ethnicity ${ }^{(17)}$

In summary, there is evidence that the majority of cancer patients would prefer to die at home and that only a small proportion of these have their preferences fulfilled. There are marked but largely unexplained variations in place of death associated with social class and other social variables. There is no research evidence on these matters in MBHA and little is known nationally about the social distribution of place of death preferences ${ }^{(2,18,19)}$. It should also be noted that average national patterns and levels of general and specialist palliative service provision and usage should not be mistaken for recommended levels of optimum care ${ }^{(1,3,17)}$.

\section{ABOUT THE STUDY}

The "Place of Cancer Deaths: Patterns and Preferences" study began at the start of October 2000, and is funded by the NHS Executive North West Research and Development Directorate for two years. Local healthcare professionals and managers will be working with academics from Lancaster and Sheffield Universities (Figure 3) to explore patterns in the place of cancer deaths, and the preferences of patients and carers for the place of terminal care in the MBHA area. Full Ethics Committee approval for this study has been obtained. The main aims of the study are outlined in Figure 4.

Dr Carol Thomas, Prof Anthony Gatrell, Dr Sara Morris: Lancaster University

Dr Malcolm Mclllmurray: Morecambe Bay Hospitals Trust

Prof David Clark: Trent Palliative Care Centre, Sheffield

Mr Timothy Mansfield: Burnley Primary Care Group, Burnley

Mrs Ann Steele: Morecambe Bay Health Authority

Mrs Chris McCann, Mrs. Mary Holland: Morecambe Bay Community Trust

Mrs Marian Whittam: St. Mary's Hospice, Ulverston

$$
\text { Figure } 3 \text { The research team }
$$

\section{PLAN OF INVESTIGATION}

In this under-researched area our approach is twofold: to build on existing research designs by repeating two studies reported in the medical/palliative care literature ${ }^{(10,16)}$ and to develop these studies in new directions as appropriate. The fact that the MBHA area has an unusual and internally variable configuration of hospice and other palliative care services
1) To gain an understanding of the unusual profile of 'place of death' patterns for cancer patients in the MBHA area

2) To describe and explain how decisions, events and circumstances interact to determine actual place of death for cancer patients in the MBHA area

3) To identify the preferences of terminally ill cancer patients and their informal carers in the MBHA area regarding the place of final care and death

4) To provide information which can assist in the further development of a palliative care strategy by MBHA

5) To contribute to health services research in the rapidly changing field of palliative care, a field in which leading scholars acknowledge that there are many unanswered questions about patient/carer preferences, and where no single 'ideal model of care' has been identified

$$
\text { Figure } 4 \text { Main aims of the study }
$$

means that this approach will yield particularly interesting data but preserve some degree of comparability. There are three key elements in the study design, which are outlined below.

Gaining an understanding of the profile of place of death patterns for cancer patients in the MBHA area

A preliminary assessment of place of death, by locality, has already been undertaken by $\mathrm{MBHA}^{(7)}$, but the report called for further research into the determinants of place of death. Our first objective will be to model place of death using data from the Public Health Mortality File ${ }^{(16)}$. Logistic regression and other generalised linear models will be used to determine some of the influences on place of death, using covariates that come from the mortality data themselves (age, gender, tumour type) and from ancillary sources (such as the census and geodemographic indicators). This will allow us to investigate variations in access to hospice services. This research will provide a useful backdrop to the exploration of patient preferences.

Describing and explaining how decisions, events and circumstances interact to determine actual place of death in MBHA

It will be important to understand how palliative care services in the Morecambe Bay area have developed over the years. To do this we plan to conduct a 'rapid appraisal' of service provision, using readily available documentary evidence, and new data generated through interviews with selected key professionals, voluntary sector workers and community leaders. In addition to enabling us to gather data on service developments and patterns of provision, the interviews with key service providers, such as GPs and cancer support nursing staff, will enable the preferences of local 'stake holders' to be voiced. Service providers' explanations for current place of final care/death patterns in Morecambe Bay, and what they think would constitute an 'ideal' situation for patients and carers, will complement the study's other foci on patient and carer preferences.

Identifying the preferences of terminally ill cancer patients and their family/carers in MBHA regarding the place of palliative/final care and the place of death

Here we plan to conduct a very similar prospective study to that undertaken by Townsend ${ }^{(10)}$. We hope, however, to go beyond that study exploring the meanings patients and carers attach to their experiences. Protocols and systems will be set up so that the research team is informed of patients diagnosed as having cancer and whom consultants think likely to live for less than one year in the Morecambe Bay area. Among those thought likely to live for less than one year, a stratified (by 
area of residence) sample will be selected over twelve months with a view of obtaining an achieved sample of 50 cancer patients across the MBHA area. Interestingly, Townsend et al found that only a small proportion of their sample did not know their diagnosis or prognosis. One of a small number of interviewers, who have experience in interviewing the dying and bereaved, will approach those sampled at the stage when those in charge of their medical care estimate that the patient has about three months to live. Potential participants will be given written information and an opportunity to discuss what taking part will entail before they either agree or decline to take part. The researchers will travel to participants to conduct interviews. Those patients who agree to participate will be involved in a series of shorter and longer interviews, some exploring preferences, and some tracking changes in their location. A validated questionnaire will be administered along with carefully and sensitively crafted questions covering the following key areas: quality of life, levels of pain and other symptoms, what patients feel about current care arrangements, whether patients have plans for their future care. Carers who are willing will be interviewed for their assessment of the patient's care circumstances twice: whilst the patient is alive and three months after the patient's death.

\section{How this research may benefit patients, carers and health professionals}

This research project aims to provide a comprehensive picture of the patterns and preferences that surround cancer deaths in Morecambe Bay. The project offers the opportunity to explore the interrelation between user preferences, circumstances and services to understand how end of life care is shaped. Close attention to user situations and preferences will be complemented by the epidemiological mapping and professionals' understanding of services to provide a detailed picture of the factors which influence the location of terminal care and death. These data will not only be valuable for the planning of services, but offer the opportunity for both lay and professional perspectives to be considered. In seeking to understand how and why cancer deaths occur in certain locations, we hope to provide information that will indicate the most appropriate ways in which services may respond to patient and carer preferences.

The government's recent 'NHS Cancer Plan' stresses the paramount importance of providing the best possible care for dying patients and pledges an extra $£ 50$ million to end inequalities in access to specialist palliative care. The plan states that "the care of all dying patients must improve to the level of the best"(20). Cancer services, including palliative care services, are in a process of significant change. Meeting the preferences of patients and carers has been identified as centre stage. By addressing the under-researched area of patient/carer preferences for place of final care and death we aim to assist in providing valuable information of both local and wider relevance.

\section{INVITATION}

We would welcome help from any healthcare professionals who see cancer patients in the terminal phases. If you are interested in our project and feel you might like to be involved, we would be very pleased to hear from you (contact details are given in Figure 5). There are two ways in which you could participate:

- assist in the recruiting of eligible patients (diagnosed with any cancer, aged 18 or over, judged to have a year or less to live)
- offer an interview. We wish to obtain data on professionals perspectives on terminal care services and practice. If you feel you have something to say about this subject, we would like to interview you.

Dr Sara Morris, Department of Applied Social Science, Cartmel College, Lancaster University, Lancaster, LA1 4YL

Phone: 01524594126 Email: s.m.morris@lancaster.ac.uk

Figure 5 Project contact details

\section{REFERENCES}

1 Higginson I. Palliative and Terminal Care. In: A. Stevens and J. Raftery (Eds.) Health Care Needs Assessment. Second Series. Radcliffe Medical Press 1997

2 Higginson IJ, Jarman B, Astin P, Doan S. Do social factors affect where patients die? An analysis of 10 years of cancer deaths in England. J Public Health Med 1999;21(1):22-28

3 Department of Health. A Policy Framework for Commissioning Cancer Services: A report by the Expert Advisory Group on Cancer to the Chief Medical Officers of England and Wales (Calman-Hine Report). Department of Health 1995

4 Department of Health Concordat: Caring for Cancer Conference (April 1998). In: NW Region Adult Palliative Care Services Accreditation Manual (Draft) Version 1.3. May 1998

5 NHS Executive A Policy Framework for Commissioning Cancer Services: Palliative Care Services. EL (96) 85. NHSE 1996

6 NW Region Adult Palliative Care Services Accreditation Manual (Draft) Version 1.3. May 1998

7 Morecambe Bay Health Authority. Palliative Care. MBHA 1998

8 Morecambe Bay Health Authority. Strategy for the development of palliative care services. MBHA 1998

9 Office for National Statistics Mortality statistics. Review of the Registrar General on deaths in England and Wales, 19931995. The Stationery Office 1995

10 Townsend J, Frank AO, Fermont D, Dyer S, Karran O, Walgrove A et al. Terminal cancer care and patients' preference for place of death: prospective study. Br Med J 1990;301:415-17

11 Dunlop RJ, Davies RJ, Hockley JM. Preferred versus actual place of death: a hospital palliative care support team study. Palliat Med 1989;3:197-201

12 Hinton J. Which patients with terminal cancer are admitted from home care? Palliat Med 1994;8:197-210

13 Addington-Hall JM, McCarthy M. Dying from cancer: results of a national population-based investigation. Palliat Med 1995;9:295-305

14 Karlsen S, Addington-Hall J. How do cancer patients who die at home differ from those who die elsewhere? Palliat Med 1998;12(4):279-86

15 Fakhoury W, McCarthy M, Addington-Hall J. Determinants of informal caregivers' satisfaction with services for dying cancer patients. Soc Sci Med 1996;42(5):721-731

16 Sims A, Radford J, Doran K, Page H. Social class variation in place of cancer death. Pall Med 1997;11:369-373

17 Clark D, Hockley J, Ahmedzai S (Eds.) New Themes in Palliative Care. Open University Press. 1997

18 Cartwright A. Changes in life and care in the year before death 1969-1987. J Public Health Med 1991;13(2):81-7

19 Seale C. A comparison of hospice and conventional care. Soc Sci Med 1991; 32(2):147-52

20 Department of Health. The NHS cancer plan. Department of Health. 2000 